\title{
La biblioteca universitaria y su rol en los procesos de investigación: una mirada desde los servicios de información con enfoque bibliométrico en Colombia
}

\author{
Eliana Camila Arciniegas Tinjacá \\ Yury Marcela Gómez Gutiérrez \\ Universidad de los Andes - UNIANDES, Colombia \\ Orlando Gregorio-Chaviano \\ Pontificia Universidad Javeriana - JAVERIANA, Colombia.
}

\section{CASE REPORT}

\begin{abstract}
Resumen
Objetivo. A partir de la necesidad de innovar en los servicios de información que prestan en la actualidad las bibliotecas universitarias y en búsqueda del desarrollo de los servicios brindados por el Sistema de Bibliotecas de la Universidad de los Andes, Colombia, se muestra el funcionamiento básico de una Unidad Bibliométrica, tomando como caso de estudio uno de sus departamentos.

Método. Con base en la producción científica del Departamento de Física de la Universidad de Los Andes, Colombia, registrada en Web of Science (WoS), se realiza un tratamiento de la información, utilizando como metodología la teoría de unidad bibliométrica planteada en la bibliografía revisada, la cual está constituida por las fases: control de fuentes, comunicación y difusión de resultados, y la capacitación.

Resultados. La unidad bibliométrica como posible solución a las necesidades de apoyo a la investigación que debe brindar la biblioteca universitaria en la actualidad, permite tener un acercamiento integral frente a las dinámicas de la producción, evaluación y comunicación de la ciencia.

Conclusiones. La biblioteca actualmente asume un papel protagónico dentro de las universidades. Los servicios ofrecidos desde la Unidad Bibliométrica posibilitaron, además del apoyo a los procesos de investigación, ganar reconocimiento y visibilidad en la comunidad científica, así como el aumento del uso de los servicios y especialmente las solicitudes de asesoría para la investigación.
\end{abstract}

Palabras Clave

Bibliometría; Bibliotecas universitarias; Evaluación de la ciencia; Indicadores bibliométricos; Servicios de información; Unidad bibliométrica

\section{The university library and its role in research processes: a view from information services with a bibliometric approach in Colombia}

\begin{abstract}
Objective. Based on the need to innovate in the information services currently provided by university and academic libraries and in the search for the development of the services provided by the Library System of the Universidad de los Andes, Colombia, we show the basic functioning of a Bibliometric Unit, taking one of its departments as a case study.

Method. Based on the scientific production of the Department of Physics of the University of Los Andes, Colombia, registered in the Web of Science (WoS), a treatment of the information was carried out, using as a methodology the theory of the bibliometric unit proposed in the revised bibliography, which is constituted by the phases: control of sources, communication and dissemination of results, and training.

Results. The bibliometric unit as a possible solution to the research support needs that the university library should offer today, allows for an integral approach to the characteristics of the production, evaluation and communication of science.

Conclusions: The library currently has a leading role within universities. The services offered from the Bibliometric Unit made it possible, in addition to supporting the research processes, to gain recognition and visibility in the scientific community, as well as an increase in the use of services and especially requests for research advice.
\end{abstract}




\section{Introducción}

En el entorno de la Bibliotecología y la Ciencia de la Información, el área de los servicios representa unos de los pilares de la profesión, siendo este el que permite conocer y suplir las necesidades de información, por medio del contacto y la comunicación con los usuarios. A partir de esto, los servicios están condicionados por las características de la información, su volumen, la forma de enfrentar el fenómeno de su explosión y difusión, entre otros aspectos (Santos, Cabrales, Rojas, Gregorio \& Ramírez, 2002).

Por otra parte, el propio avance y desarrollo de la Ciencia de la Información ha llevado a una especialización, cada vez mayor, de las funciones de los especialistas en información y en correspondencia, de las distintas instituciones dedicadas al trabajo de la información, en especial las bibliotecas universitarias. Desde la perspectiva de la Bibliotecología, la Ciencia de la Documentación y la Ciencia de la Información, las bibliotecas universitarias han perfeccionado continuamente su enfoque de servicios, siempre apremiados por la influencia de los cambios tecnológicos y los procesos de desarrollo científico.

Desde esta perspectiva, la biblioteca universitaria cumple una misión básica de participación en las actividades de apoyo a la investigación, las cuales tienen como punto de partida la evaluación de la productividad científica y el análisis de los dominios científicos, procesos que se centran principalmente en el estudio y uso de indicadores bibliométricos que permiten visualizar las dinámicas y tendencias presentes en los resultados de investigación de las instituciones (Torres-Salinas \& Cabezas-Clavijo, 2012).

No obstante, se observan puntos críticos que intervienen en la relación existente entre la biblioteca universitaria y los procesos de investigación, donde convergen las políticas científicas y el desarrollo a nivel internacional de servicios bibliométricos, tanto en metodologías como en nuevos indicadores. Es aquí donde el rol de la biblioteca universitaria y las nuevas propuestas conceptuales, que se desarrollan en torno al proceso de apoyo a la investigación, se hacen relevantes. Los medios para desarrollar estas propuestas son los servicios bibliométricos, por médio de indicadores, fuentes de datos, metodologias, lo cual permite contar con un insumo significativo para el análisis de la ciencia desde de diferentes fuentes de información.

\subsection{La evaluación de la ciencia desde la bibliometría}

Em la actualidad, una de las principales características de las universidades se enfoca en los procesos de evaluación y gestión de la ciencia (Alfaro, 2015), de una parte, el aporte relacionado al trabajo con herramientas de publicación de artículos científicos de impacto y de otra, la evaluación de los resultados teniendo en cuenta que la investigación se ha transformado en uno de los principales activos del sistema universitario.

La tendencia actual de evaluación de la ciencia se ve reflejada en diferentes mecanismos de medición como la evaluación del profesorado, de revistas científicas y elaboración de rankings, entre otros, donde las bases de datos de citación como Web of Science (WoS) y Scopus además de otras fuentes bibliográficas, ofrecen insumos para dicha medición y análisis lo cual se ha convertido en un elemento vital en la gestión de la investigación universitaria.

La bibliometría como herramienta de medición y que aporta indicadores (Glänzel, 2012), ha generado un fuerte impacto en las universidades, dado que a partir de los resultados que los propios indicadores aportan, se establecen muchas de las políticas que median el proceso investigativo, como por ejemplo el proceso de financiación de las investigaciones. Estas políticas son desarrolladas en su gran mayoría por las vicerrectorías de investigación que tienen como función coordinar y dirigir los procesos investigativos universitarios a partir de 
actividades de seguimiento y recursos para su desarrollo, con el fin de respaldar la productividad científica fortaleciendo y permitiendo que estos procesos sean de alto nivel (Villaveces et al., 2010).

La importancia de la bibliometría en los procesos de evaluación de la ciencia, en el desarrollo de la actividad científica actual (referente a la visibilidad, el impacto y los procesos de financiación), al igual que su relación con la ciencia de la información y la bibliotecología; ha propiciado el desarrollo de servicios bibliométricos como las unidades de bibliometría, término utilizado por Torres-Salinas y Jiménez-Contreras (2012) el cual recoge conceptualmente el establecimiento de estos servicios dentro de la biblioteca universitaria.

Dichos procesos se encuentran directamente relacionados con la bibliometría (Ball y Tunger, 2006), siendo esta un método cuantitativo y cualitativo dirigido al análisis de la producción científica universitaria llegando a convertirse, por su importancia y relación directa con los recursos de información, en uno de los nuevos focos de servicio para la biblioteca universitaria.

A pesar de ello, el papel de la mayoría de unidades de investigación dentro de las universidades y en especial las bibliotecas, no se enfocan en generar servicios de asesoría para el correcto diseño de políticas científicas y aumento de la visibilidad, servicios que podrían consolidar una metodología que permita posibilitar la creación de análisis situacionales de la institución, identificando tendencias y ofreciendo alternativas para mejorar los procesos investigativos.

\subsection{La biblioteca universitaria actual y su rol en los procesos de investigación}

El entorno cambiante que ha generado el desarrollo de las tecnologías de la información, la universalidad del conocimiento, la competitividad entre instituciones, los rankings, la visibilidad y el impacto de la actividad científica (Tovar-Sanz, 2015), han hecho que sea necesario el mejoramiento de la investigación y con ello la evolución en el rol de la biblioteca universitaria como sistema de apoyo a este proceso misional universitario, trascendiendo más allá de la institución a la cual presta sus servicios, impactando diferentes esferas sociales.

El desarrollo de la investigación en cualquier contexto se encuentra directamente relacionada con su proceso de evaluación. Al respecto, uno de sus aspectos relevantes es valorar sus resultados en diferentes agregaciones. Para ello se emplean indicadores de I+D y bibliométricos. En el marco de la evaluación bibliométrica de la investigación podemos encontrar según Glänzel (2012) tres niveles de agregación: nivel micro (autores e investigadores), nivel meso (instituciones y áreas de conocimiento) y nivel macro (países y regiones). De acuerdo con esto, la evaluación de las instituciones universitarias (nivel meso, aunque en algunos casos se toma como macro), tiene como principal insumo los resultados de investigación y desarrollo de la productividad científica que se evidencian a través de las publicaciones generadas por sus diferentes unidades.

Por lo anterior, es necesario encontrar nuevas vías para vincular la biblioteca con la universidad y sus respectivas áreas misionales, como por ejemplo el área de investigación (Maceviciute, 2014). A través de la renovación y puesta en marcha de nuevos servicios de información como búsqueda y evaluación de información, pautas para la escritura de artículos científicos de impacto, selección de revistas para publicar, uso de gestores bibliográficos, entre otros, la biblioteca puede brindar apoyo y generar un proceso de comunicación con sus usuarios y a su vez con los procesos actuales de educación, lo cual permitirá mejorar el quehacer universitario de la institución.

El liderazgo de la biblioteca en el apoyo a la investigación (Åström \& Hansson, 2013), se ve también reflejado en la necesidad de dar acceso y preservar la producción investigativa universitaria, principales responsabilidades desde sus inicios. Por el contrario, los procesos de evaluación de la producción científica de las instituciones de 
educación superior, como los conocemos hoy en día son más recientes y emergieron apenas en el siglo pasado (Chinchilla-Rodríguez, 2004).

Sin embargo, lo nuevos conceptos y enfoques presentes en la biblioteca universitaria no ofrecen las soluciones necesarias a la mayoría de los procesos de investigación, sino que se reducen únicamente al tratamiento y capacitación en herramientas de producción científica, como lo son las bases de datos de análisis de citas, la obtención de documentos y la referencia especializada como se evidencia en la teoría de Pinto, Sales y Osorio (2008). No obstante, como ya se ha mencionado, la bibliometría, sus indicadores y metodologías de trabajo, pueden ser de utilidad en el mejoramiento de los servicios de información y en la posibilidad de eliminar las limitaciones existentes.

De acuerdo con las limitaciones evidenciadas en el tratamiento de los servicios bibliométricos a nivel mundial, entre ellas se encuentran diversos estudios (Corrall, Kennan, Afzal \& Sen, 2013; Iribarren-Maestro, Grandal, Alecha, Nieva, San-Julián, 2015; Torres-Salinas \& Jiménez-Contreras, 2012), los cuales cuestionan el modo en el que se teoriza y orienta la práctica en estos servicios dentro de las bibliotecas universitarias. A partir del desarrollo del área bibliométrica y la importancia que tiene la aplicación de ésta en las universidades alrededor del mundo, se propone una visión de unidad bibliométrica en la cual los servicios bibliométricos se articulen y puedan gestionarse con el fin de garantizar una asistencia integrada y de calidad que verdaderamente ayude al área de investigación en su quehacer misional.

La publicación de artículos científicos, como insumo fundamental dentro del proceso de investigación, se ha convertido en la unidad estándar para calificar la calidad del proceso generador de conocimiento y su impacto en el entorno científico (Rueda-Clausen Gómez, Villa-Roel \& Rueda-Clausen Pinzón, 2005), de esta manera, los servicios bibliométricos cobran relevancia dentro de la biblioteca universitaria, cambiando el paradigma reduccionista de la biblioteca como proveedora de contenidos y recursos de información. El personal especializado ha empezado a responder a los requerimientos anteriormente citados con servicios de innovación como los bibliométricos, permitiendo a la biblioteca universitaria abordar diferentes formas de apoyo a la investigación, como capacitaciones en el uso de herramientas de medición de productividad científica, apoyo en la difusión de la investigación, asesoramiento en la selección de revistas para publicar, entre otros.

Pese a la escasa cantidad de servicios especializados, se observan adelantos importantes en el apoyo a la investigación por parte de la biblioteca universitaria, aunque también se evidencia que existen diferentes limitantes en el manejo de dichos servicios y su implementación se muestra aislada, ya que no permite unir resultados de diferentes instituciones y por ende no cuenta con una metodología claramente establecida para ofrecer dichos servicios. En este estudio se muestran los principales servicios con los que debería contar la biblioteca universitaria para apoyar la investigación como la formación de usuarios, la orientación bibliométrica, desarrollo de repositorios institucionales, búsquedas especializadas, ayuda al profesorado en la preparación de perfiles, entre otros.

En conclusión, la biblioteca actual debe responder a las necesidades de los investigadores con diversos servicios de apoyo a sus actividades, entre los que se encuentran: el uso de la bibliometría y sus servicios, indicadores y metodologías que se conviertan en fuente de evaluación de la investigación desarrollada en la universidad (Johnson, Butler \& Johnston, 2012)

De esta manera, dichas unidades entienden la importancia de apoyar la creación de conocimiento e investigación por medio de sus servicios. En general, evidencian tener servicios orientados únicamente a formar usuarios en el uso de bases de datos, gestores bibliográficos, pero aún se encuentran alejadas de ofrecer un 
apoyo integral a la investigación que se adapte a las necesidades de los investigadores, ofreciendo asesoramiento en el uso estratégico de los recursos de información para la investigación y fortaleciendo la visibilidad de la investigación institucional.

\subsection{Las unidades de bibliometría como apoyo a la visibilidad de la investigación}

Las unidades de bibliometría o unidades bibliométricas agrupan servicios bibliométricos que responden a tres pilares fundamentales enfocados de forma integral a la relación entre la biblioteca y el desarrollo investigativo:

(1) Control de las fuentes información sobre investigación (a través de las bases de datos de actividad científica institucional), (2) Generación de informes de análisis, prospectiva y vigilancia, (3) Formación, asesoramiento y consulta experta (Torres-Salinas \& Jiménez-Contreras, 2012).

Para dichas unidades, el establecimiento de un sistema de información centralizado que permita gestionar toda la producción científica institucional es de vital importancia, con el fin de obtener la información adecuada y necesaria que genere los indicadores bibliométricos para el tratamiento de los datos de investigación de los diferentes sistemas tanto internos, como externos a la universidad. Las mismas establecen como herramientas, la formación de sistemas de información científica, suites bibliométricas de compañías comerciales, la adopción de rankings de universidades, entre otros.

En cuanto al pilar de formación, asesoramiento y consulta experta, la bibliometría ofrece insumos que sirven como herramienta de medición y supervisión para el análisis de la producción científica. Es importante entonces que desde la biblioteca universitaria se adopte un papel académico o formativo que apoye por medio de la capacitación y el asesoramiento al cuerpo investigativo, las actividades investigativas en pro de seguir las tendencias y los desafíos internacionales que exigen calidad de producción científica universitaria.

La asesoría y capacitación apuntan al incremento de casos exitosos de proyectos de investigación científica, de financiamiento para la investigación al igual que el reconocimiento de grupos de investigación, de manera personalizada, así como contextualizar y apropiar a los investigadores con conceptos bibliométricos, generando un acercamiento teórico que permita identificar, conocer y utilizar indicadores, índices y herramientas de análisis de información, de manera grupal o en cursos. Ambos enfoques de formación pretenden mejorar las buenas prácticas de comunicación de la ciencia.

En Colombia se evidencia que las bibliotecas universitarias no poseen un avance significativo en el área de servicios que apoyen la investigación, en especial servicios bibliométricos. De diez bibliotecas analizadas, cinco poseen al menos un servicio caracterizado como servicio de apoyo a la investigación. Las Universidad de Antioquia, Nacional, Andes, Rosario y Universidad Pontificia Bolivariana ofrecen servicios de asesoría especializada, trabajo con bases de datos especializadas, gestores bibliográficos, apoyo a los procesos de investigación, entre otros, pero no un servicio que de forma integral y desde el punto de vista metodológico y práctico incluya herramientas y procesos de una unidad bibliométrica.

La presente investigación expone las tendencias y perspectivas actuales en torno al desarrollo y funcionamiento de los servicios de información para la investigación desde la perspectiva bibliométrica. A partir de la descripción general del rol que debe jugar el profesional de la información en la actualidad y dados los cambios que operan en las bibliotecas, la necesidad de pasar de servicios tradicionales a servicios innovadores es imperante. Por lo que para mostrar el funcionamiento del servicio referenciado, se presenta el funcionamiento en la Sistema de Bibliotecas de la Universidad de Los Andes, Bogotá, Colombia, específicamente servicios bibliométricos a partir de la evaluación del Departamento de Física y sus resultados en Web of Science (WoS). 


\section{Material y métodos}

Para la propuesta metodológica de la presente investigación se tomó una muestra de la producción científica del Departamento de Física de la Universidad de Los Andes, Colombia, a partir de la información registrada en Web of Science (WoS). Como parámetros de búsqueda se utilizó un rango de tiempo de 15 años, en el área de investigación Física y tipologías documentales citables (artículos, revisiones y cartas), para obtener 1080 documentos, lo que permitió tener una visión general de las dinámicas de publicación evidenciadas y mostrar los principales aspectos metodológicos del funcionamiento de una unidad bibliométrica en una universidad, de acuerdo con los planteamientos de la bibliografía especializada.

Se partió del control de fuentes como primera fase o eje de trabajo, no obstante, es preciso mencionar que para este caso de estudio no se realizó un proceso de control, unificación y normalización de fuentes, ya que se utilizó una única fuente de información desde la cual se generaron los indicadores bibliométricos (WoS).

Para la generación de informes, segundo eje de la unidad se tomó como insumo principal la información obtenida del análisis de resultados, seleccionando los indicadores más representativos y combinando variables que mostraran detalladamente la producción investigativa del Departamento de Física de la Universidad de los Andes. Como herramientas de comunicación y difusión se plantean 3 tipos de informes, dos de ellos llamados Flash Bibliométicos, enfocados a trabajar en dos líneas: la primera, mostrar de manera resumida la productividad del Departamento con respecto a la Universidad y la segunda, exponer cuáles eran las redes de colaboración evidenciadas. El tercer informe, muestra datos bibliométricos especializados para estudiar factores de liderazgo, producción, visibilidad, financiación y colaboración de la unidad analizada.

Por último, en el eje de capacitación y asesoría, se formulan dos fichas de capacitación que muestran los objetivos, lineamientos y contenidos necesarios para llevar a cabo una formación sobre principios teóricos y prácticas bibliométricas para diferentes agregados y se proponen indicadores que permitan realizar análisis y obtener conclusiones sobre las capacitaciones ofrecidas para posibles mejoras.

\section{Estructura del servicio}

Dado que los indicadores bibliométricos son herramientas válidas para la gestión de las universidades y las unidades que la conforman al detectar fortalezas y debilidades y posibilitar la correcta gestión en la toma de decisiones, contar con herramientas, fuentes y bases de datos que de manera precisa e inmediata aporten indicadores para la evaluación y el diseño de políticas científicas es un elemento esencial.

Por tanto, se presentan las diferentes fases de la unidad como servicio de información que presta la Biblioteca de la Universidad de Los Andes y en cada una de ellas los indicadores, metodologías e informes válidos para la correcta gestión de la actividad científica en el departamento que ha servido como insumo para mostrar el funcionamiento.

\subsection{Fases de la propuesta de servicio en unidad bibliométrica}

\section{Fase 1: Control de fuentes e Indicadores bibliométricos}

A partir del análisis de la información recuperada y evaluada, se presentan los resultados en función del control de fuentes como primera fase o eje de trabajo de la Unidad Bibliométrica. El control de fuentes posibilita a partir de la sistematización de la información producida por las dependencias universitarias, la generación de 
indicadores para la toma de decisiones y el diseño de políticas científicas en diferentes niveles de agregación, lo que lleva además al aumento de la visibilidad y de modo específico la importancia para el análisis de la clasificación de grupos.

En el análisis del Departamento de Física de la Universidad de Los Andes y a partir de la fase 1 se muestran los principales resultados de su actividad científica lo cual posibilita la evaluación para la toma de decisiones y el diseño de políticas científicas futuras. De igual forma, los resultados de esta fase posibilitan el desarrollo de las siguientes, en especial la generación de indicadores y la capacitación.

Los indicadores obtenidos a partir de la producción científica, más allá del análisis bibliométrico tiene como finalidad la creación de Datasets de indicadores con los resultados de la actividad científica, los cuales se entregan al propio departamento y forman parte además del sistema de información de la biblioteca con los cuales se trabaja en la medida de las necesidades y pueden formar parte además de las memorias institucionales en caso de que se requieran.

\subsection{Dataset de indicadores del Departamento de Física de la Universidad de Los Andes, Bogotá, Colombia.}

Los Datasets constituyen agrupaciones de indicadores sobre el agregado evaluado, de forma tal que sea posible la generación de los mismos para la toma de decisiones en diferentes procesos al interior de la universidad (diseño de políticas científicas, participación en convocatorias, planeación de la investigación, entre otros). En este caso se presentan en las siguientes tablas, figuras e ilustraciones), aquellos análisis representativos para el servicio planteado. Indicadores de productividad, citación y colaboración, entre otros, los cuales aportan información sobre el comportamiento de la actividad científica del departamento.

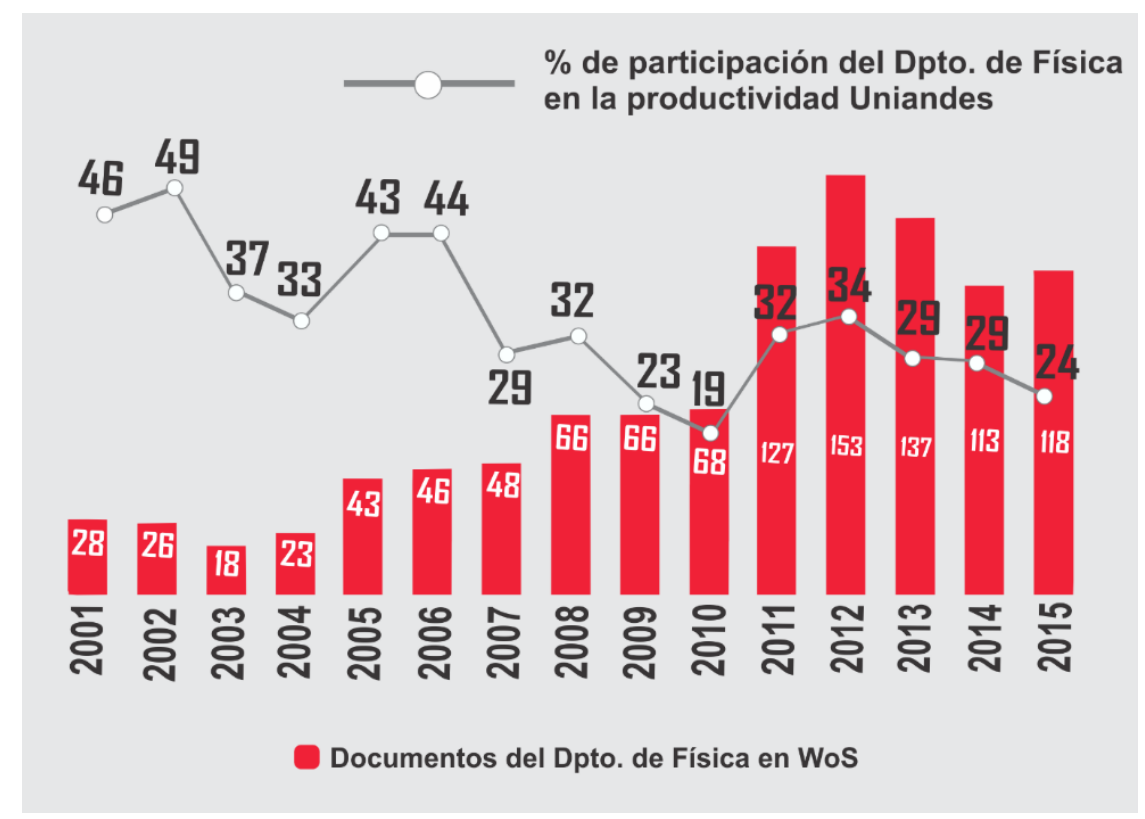

Figura 1 - Porcentaje de participación del Departamento de Física en la productividad de la universidad y número de documentos en el periodo 2001-2015. 


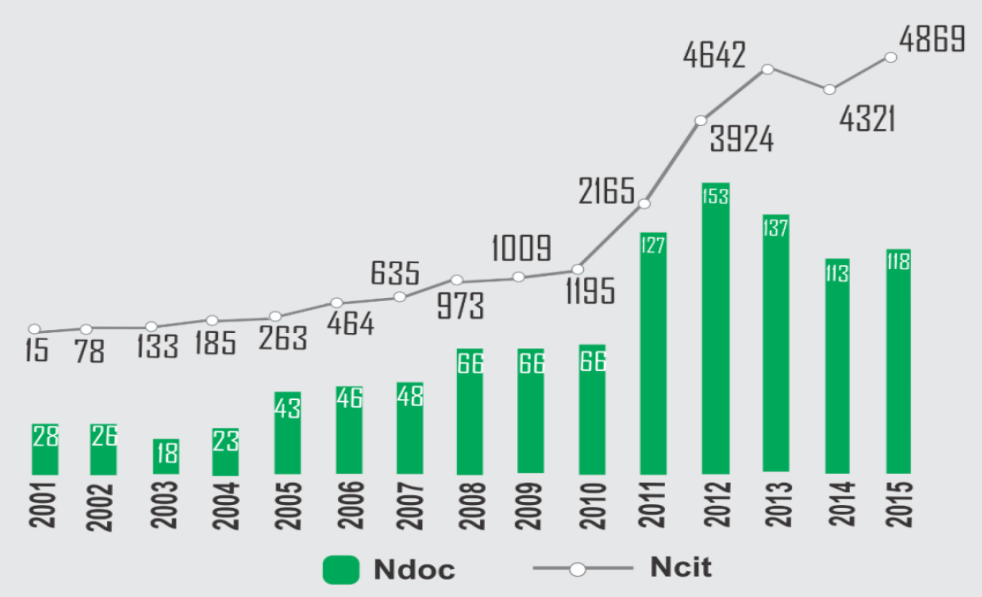

Figura 2 - Número de documentos y citas entre 2001-2015.

Fuente: Elaborado por el autor

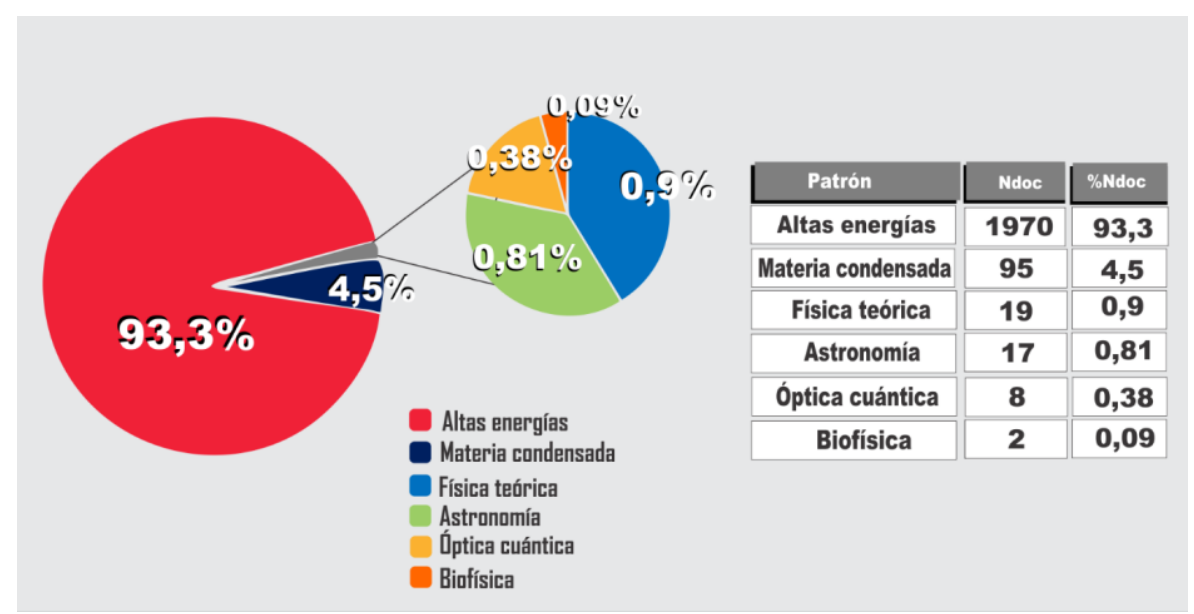

Figura 3 - Porcentaje de documentos por grupo de investigación en el departamento. Fuente: Elaborado por el autor

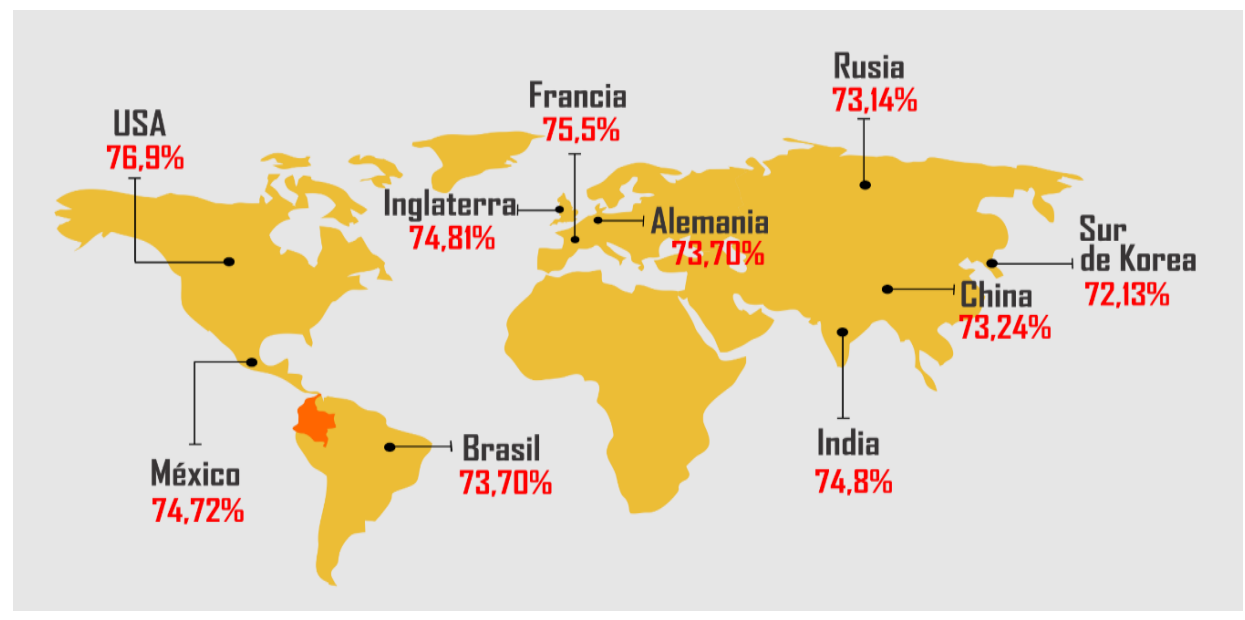

Ilustración 1 Principales países que trabajan en colaboración con el Departamento de Física. Fuente: Elaborado por el autor 


\begin{tabular}{|c|c|c|}
\hline Patrón & $\begin{array}{c}\text { \%Ndoc } \\
\text { Internacional }\end{array}$ & $\begin{array}{c}\text { \%Ndoc } \\
\text { Nacional }\end{array}$ \\
\hline Internacional & $\mathbf{9 3 9}$ & $\mathbf{9 1 , 7}$ \\
\hline \hline Nacional & $\mathbf{2 0}$ & $\mathbf{2}$ \\
\hline \hline Institucional & $\mathbf{3 2}$ & $\mathbf{3 , 1}$ \\
\hline Sin colaboración & $\mathbf{3 3}$ & $\mathbf{3 , 2}$ \\
\hline
\end{tabular}

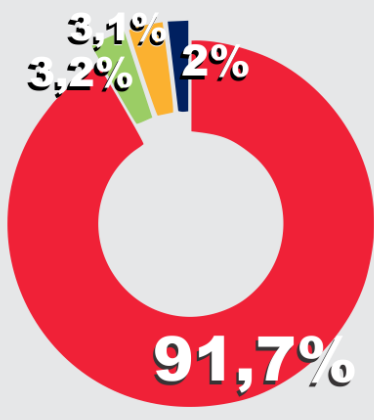

Figura 4 - Patrones de colaboración y liderazgo del el Departamento Fuente: Elaborado por el autor

\begin{tabular}{|c|c|c|} 
Liderazgo & $\begin{array}{c}\% \text { Ndoc } \\
\text { Internacional }\end{array}$ & $\begin{array}{c}\text { \%Ndoc } \\
\text { Nacional }\end{array}$ \\
\hline \hline SI & $\mathbf{6 , 3 9}$ & $\mathbf{7 0}$ \\
\hline \hline NO & $\mathbf{9 3 , 6 1}$ & $\mathbf{3 0}$ \\
\hline
\end{tabular}

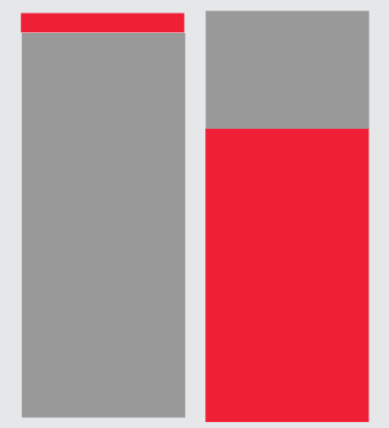

INTERNACIDNAL NACIDNAL

Liderazgo

No liderazgo

\begin{tabular}{|c|l|c|c|c|c|c|c|c|}
\hline No & \multicolumn{1}{|c|}{ Revista } & Ndoc & $\%$ Ndoc & Ncit & Categoria 1 & FI & FIN & Q \\
\hline 1 & Physical Review Letters & 236 & $25 \%$ & 7997 & Physics, Multidisciplinary & 7.51 & 2.54 & 1 \\
\hline 2 & Physics Letters B & 197 & $20 \%$ & 8807 & Physics, Multidisciplinary & 6.13 & 2.07 & 1 \\
\hline 3 & Physical Review D & 183 & $19 \%$ & 3505 & Astronomy \& Astrophysics & 4.64 & 1.39 & 1 \\
\hline 4 & $\begin{array}{l}\text { Journal of High Energy } \\
\text { Physics }\end{array}$ & 134 & $14 \%$ & 1660 & Physics, Particles \& Fields & 6.11 & 3.17 & 1 \\
\hline 5 & $\begin{array}{l}\text { The European Physical } \\
\text { Journal C }\end{array}$ & 54 & $6 \%$ & 1074 & Physics, Particles \& Fields & 5.08 & 2.64 & 1 \\
\hline 6 & Physical Review E & 23 & $3 \%$ & 349 & Physics, Mathematical & 2.29 & 7.35 & 1 \\
\hline 7 & Physical Review A & 15 & $2 \%$ & 337 & Optics & 2.81 & 0.87 & 1 \\
\hline 8 & $\begin{array}{l}\text { Journal of Statistical } \\
\text { Physics }\end{array}$ & 15 & $2 \%$ & 49 & Physics, Mathematical & 1.2 & 3.86 & 3 \\
\hline 9 & Nucl Instrum Meth A & 14 & $2 \%$ & 691 & Nuclear Science \& & 1.22 & 5.03 & 2 \\
\hline 10 & Physical Review C & 12 & $1 \%$ & 424 & Physics, Nuclear & 3.73 & 3.32 & 1 \\
\hline
\end{tabular}

Tabla 1 - Patrones de colaboración y liderazgo del el Departamento.

Fuente: Elaborado por el autor 


\begin{tabular}{|l|l|c|c|c|c|}
\hline \multicolumn{1}{|c|}{ institución } & \multicolumn{1}{c|}{ País } & Noc & \%Ndoc & Ncit & \%Nocit \\
\hline Centro Nacional para la Investigación & Francia & 806 & 74.6 & 23.971 & 92.4 \\
\hline Universidad de Chicago & Estados Unidos & 791 & 73.2 & 23.820 & 91.6 \\
\hline Universidad de California & Estados Unidos & 790 & 73.1 & 23.749 & 91.6 \\
\hline $\begin{array}{l}\text { Departamento de Energía de los Estados } \\
\text { Unidos }\end{array}$ & Estados Unidos & 790 & 73.1 & 23.815 & 91.8 \\
\hline Laboratorio Nacional Fermi & Estados Unidos & 788 & 72.9 & 24.161 & 91.8 \\
\hline Instituto de Física de Alta Energía & China & 785 & 72.6 & 23.796 & 91.8 \\
\hline Universidad Panjab & India & 785 & 72.6 & 23.704 & 91.4 \\
\hline Universidad de Notre Dame & Estados Unidos & 785 & 72.6 & 23.683 & 91.6 \\
\hline Universidad Estatal de Florida & Estados Unidos & 784 & 72.3 & 23.752 & 91.6 \\
\hline Universidad Estatal de Moscú & Rusia & 784 & 72.5 & 23.754 & 91.6 \\
\hline Universidad Rice & Estados Unidos & 784 & 72.5 & 23.651 & 91.2 \\
\hline Universidad Estatal de Nueva York & Estados Unidos & 784 & 72.5 & 23.678 & 91.3 \\
\hline Universidad de Kansas & Estados Unidos & 784 & 72.5 & 23.645 & 91.2 \\
\hline
\end{tabular}

Tabla 2 - Instituciones y países que colaboran con el Departamento de Física de la Universidad de Los Andes.

Fuente: Elaborado por el autor

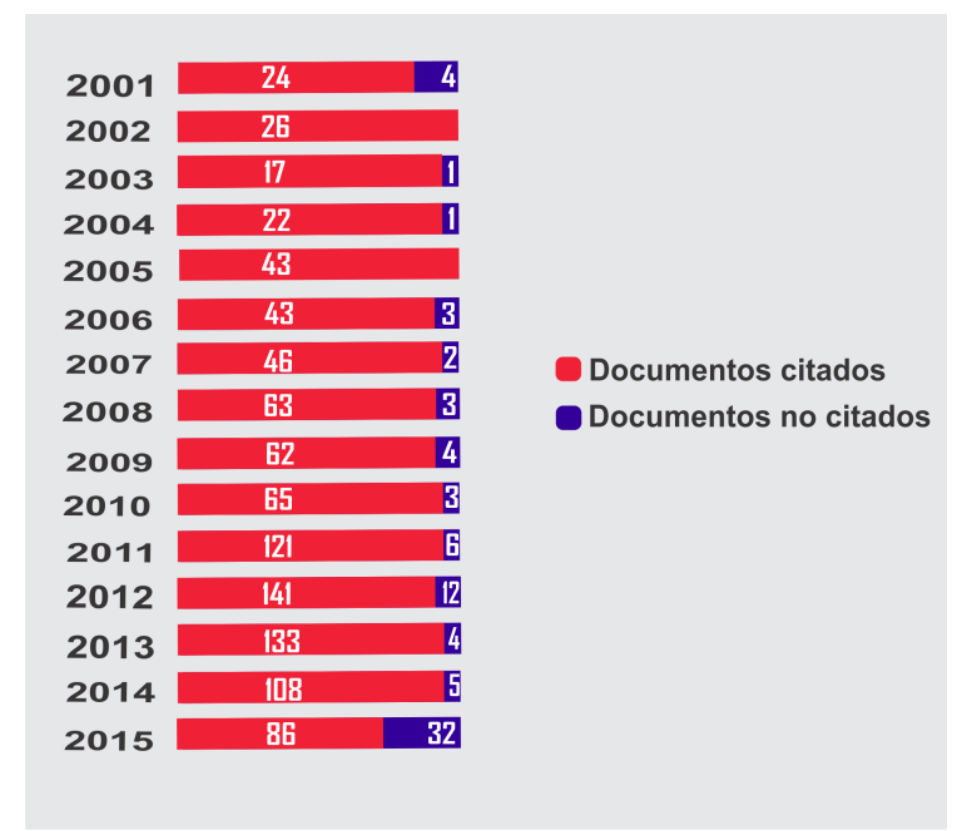

Figura 5. Relación entre documentos citados y no citados por año en la actividad científica del departamento.

Fuente: Elaborado por el autor 


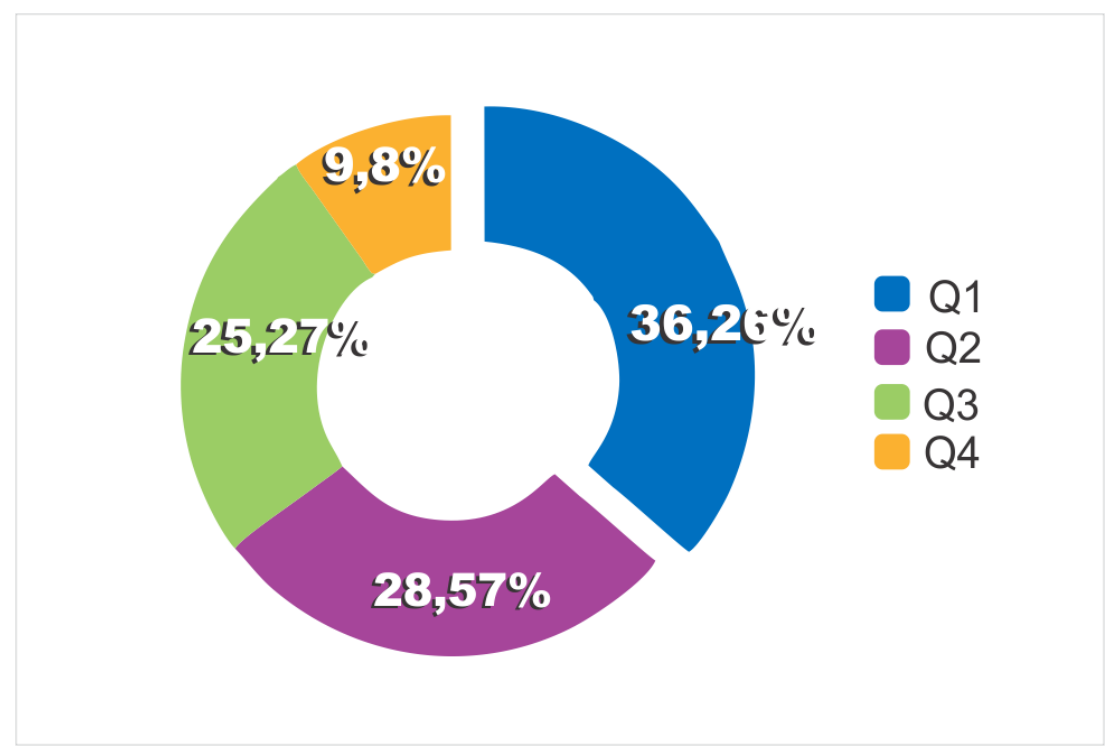

Figura 6. Producción absoluta y porcentual por cuartiles. Fuente: Elaborado por el autor

\begin{tabular}{|l|c|c|}
\hline Patrón & \% Ndoc Internacional & \% Ndoc Nacional \\
\hline Internacional & 939 & 91.7 \\
\hline Nacional & 20 & 2 \\
\hline Institucional & 32 & 3.1 \\
\hline Sin colaboración & 33 & 3.2 \\
\hline
\end{tabular}

Tabla 3. Patrones de colaboración en el Departamento de Física. Fuente: Elaborado por el autor

\section{Fase 2: Comunicación y difusión de resultados}

La difusión de los resultados de la actividad científica en diferentes niveles de agregación es un aspecto clave que posibilita no solo que el entorno de la universidad conozca los resultados y aquellas dependencias de mayor visibilidad e impacto, sino también motiva y fortalece el trabajo investigativo en las dependencias. En las bibliotecas o unidades de información aporta visibilidad en el entorno universitario y permite que el servicio vaya madurando al ser conocido por la comunidad.

En la Unidad Bibliométrica de la Universidad de Los Andes y en el trabajo específico con el Departamento de Física, se creó un medio de difusión llamado flash informativo el cual cuenta con resúmenes de resultados e indicadores de su actividad científica. Como se observa en las imágenes, esta fuente de información permite la visualización en el contexto universitario a partir de diferentes fuentes (tableros, redes sociales, etc.), de aquellas métricas que muestran el comportamiento de las unidades y de la universidad en general, de forma sencilla y resumida. De igual forma se realizan rankings de departamentos, facultades y autores a partir de indicadores como el Indice h y se ofrece información general de las dependencias, los cuales se muestran en tableros de anuncios. 


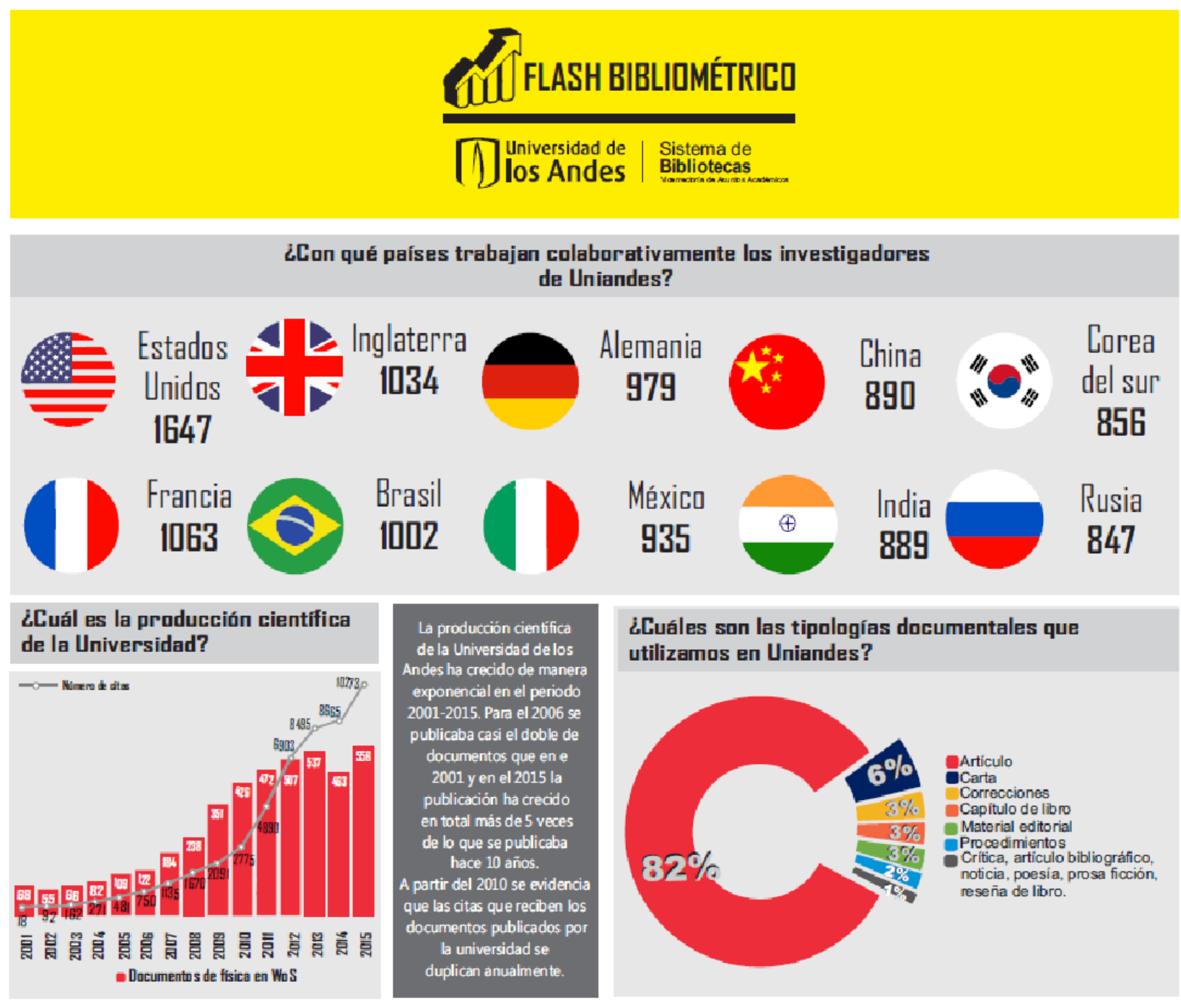

Ilustración 2. Producción, colaboración y tipologías documentales representativas de la universidad.

Fuente: Elaborado por el autor 


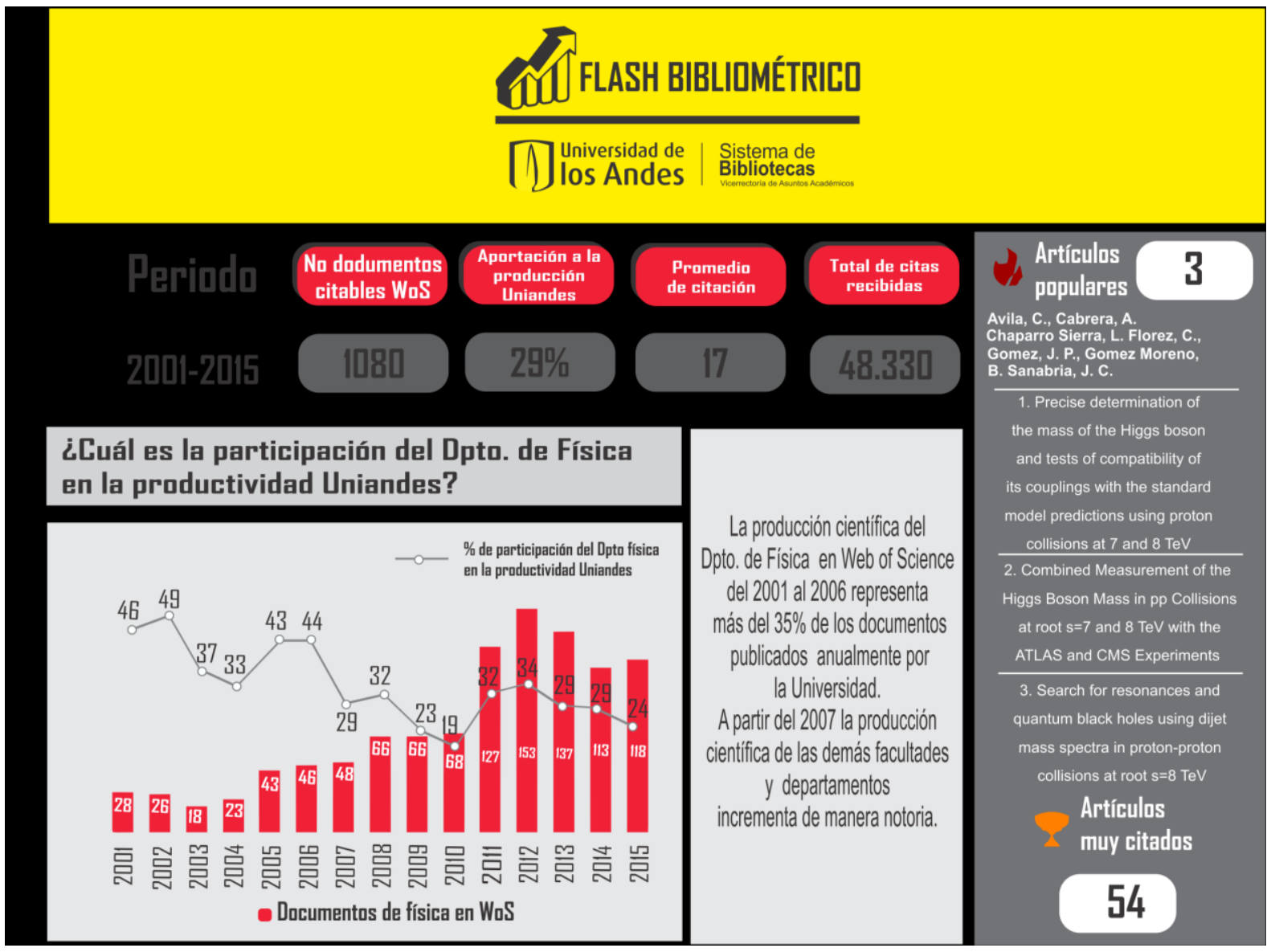

Ilustración 3. Participación total y porcentual del departamento en la actividad científica de la Universidad.

Fuente: Elaborado por el autor

\section{Fase 3: Capacitación}

El módulo o fase de capacitación del servicio tiene como principal objetivo fortalecer las habilidades en función del uso de fuentes de datos en la gestión de la investigación y, por tanto, propiciar la visibilidad de la actividad científica. Más allá de las búsquedas de información y acompañamiento que la biblioteca realiza a la comunidad, se ofrecen servicios más avanzados como búsqueda de información con fines estratégicos (gestión de proyectos de investigación), aspectos generales de la bibliometría como herramienta de evaluación, el análisis de la información resultante a partir de indicadores bibliométricos y la escritura de artículos científicos de impacto, la puesta en práctica de estrategias de difusión de los resultados, el mejoramiento de las revistas institucionales, entre otros.

Para cada servicio se crea una ficha con objetivos, alcance, perfiles de usuarios, descripción general del servicio, contenidos e indicadores, de forma tal que pueda llevarse un control sobre el uso, pertinencia e interés general del mismo dentro de la comunidad universitaria.

A partir del análisis de la producción científica de las diferentes dependencias se seleccionan aquellas que más necesitan de los referidos servicios especializados (en este caso la capacitación) y se evalúa su desempeño en el tiempo, antes y después de las capacitaciones a partir de sus publicaciones, participación en congresos, etc. 


\begin{tabular}{|c|c|}
\hline \multicolumn{2}{|r|}{ FICHA ASESORÍA \# 1} \\
\hline \multicolumn{2}{|c|}{ ¿CÓMO MEJORAR SU VISIBILIDAD EN FUENTES DE INFORMACIÓN? } \\
\hline Objetivo & $\begin{array}{l}\text { Brindar asesoría en técnicas de comunicación de la producción } \\
\text { científica, a través del estudio de casos específicos, con el fin de } \\
\text { mejorar la visibilidad de los resultados científicos del investigador. }\end{array}$ \\
\hline Alcance & $\begin{array}{l}\text { Inicia con la solicitud de la capacitación por parte del usuario y finaliza } \\
\text { con la evaluación de la capacitación después de realizarla. }\end{array}$ \\
\hline Perfil de usuario & $\begin{array}{ll} & \text { Docentes universitarios } \\
\text { - } & \text { Investigadores } \\
\end{array}$ \\
\hline Lineamientos & $\begin{array}{l}\text { - La asesoría debe ser ofrecida por un especialista en temas } \\
\text { bibliométricos que domine las temáticas a tratar (véase: perfil del } \\
\text { especialista). } \\
\text { - El especialista debe tener conocimiento de las dinámicas de } \\
\text { publicación. } \\
\text { - La asesoría debe brindar información actualizada a los usuarios. } \\
\text { - De acuerdo a la especificidad de la solicitud se ofrecerá } \\
\text { capacitación o asesoramiento. }\end{array}$ \\
\hline $\begin{array}{l}\text { Descripción de } \\
\text { contenidos }\end{array}$ & $\begin{array}{l}\text { - Análisis de la producción cientifica del agregado analizado. } \\
\text { - Normalización y unificación de firmas y filiación en diferentes } \\
\text { fuentes. } \\
\text { - Creación del ORCiD. } \\
\text { - Gestión de perfiles en fuentes alternativas. } \\
\text { - Tácticas para la presentación de un artículo científico de alto } \\
\text { impacto. } \\
\text { - Recomendaciones y/o sugerencias de mejoramiento de la } \\
\text { visibilidad. }\end{array}$ \\
\hline \multirow[t]{3}{*}{ Indicador } & \\
\hline & \# asesorías \\
\hline & [Total de usuarios del agregado] \\
\hline
\end{tabular}

Figura 7. Modelo de ficha de capacitación. Fuente: Elaborado por el autor 


\section{Consideraciones finales}

Los servicios de información ofrecidos en la biblioteca universitaria actual deben enfocarse a satisfacer las necesidades de los usuarios, especialmente de los investigadores que buscan encontrar en ella herramientas útiles para el desarrollo de sus actividades de investigación, razón por la cual deben abarcar deben abarcar diversas áreas de aplicación, entre ellas la bibliometría, ofreciendo servicios innovadores donde la biblioteca sea participe de los procesos de investigación.

La bibliometría y los indicadores que esta aporta, constituyen elementos esenciales tanto en la evaluación y mejoramiento de la visibilidad de las instituciones como en el aporte de metodologías de trabajo enfocadas a los servicios de información y donde la biblioteca universitaria puede tener un liderazgo significativo brindando servicios de información innovadores. Es necesario fortalecer el papel de la biblioteca como creadora de conocimiento, fuentes de información, metodologías de investigación y trabajo con indicadores bibliométricos, que ofrezca la posibilidad (y la necesidad) de trabajar junto a las dependencias y departamentos de la universidad; ello posibilitará ganar espacios de reconocimiento, visibilidad institucional y mejoramiento de los procesos de investigación. También, la contribución de la biblioteca y los profesionales de la información en la búsqueda de indicios de calidad para la evaluación de las diferentes dependencias universitarias y claro está de los grupos de investigación y profesores es clave.

En el caso de los servicios que se ofrecen desde la Unidad Bibliométrica en el Sistema de Bibliotecas de la Universidad de Los Andes y su funcionamiento a partir de una muestra, se pudo comprobar no solo la importancia de los mismos para el mejoramiento de la visibilidad y el impacto de las dependencias universitarias, sino como la biblioteca puede jugar un papel importante a la vez que obtiene liderazgo en el entorno de la universidad.

Las tres fases de trabajo en las que se insertan los servicios ofrecidos por la Biblioteca de la Universidad de Los Andes, permitieron pasar de la prestación de servicios tradicionales (búsqueda de información en bases de datos, referencia, préstamo de materiales bibliográficos, etc.) a servicios para la investigación. Ello ha posibilitado no solo ganar reconocimiento y ser reconocidos dentro de la comunidad, sino aumentar el uso de la biblioteca y especialmente las solicitudes de asesoría en la evaluación y gestión de la investigación.

\section{Referencias}

Alfaro, P. (2015). La biblioteca universitaria como soporte a la investigación: la importancia de los rankings universitarios. Revista de Unidades de Información, (8), 1-34.

Åström, F., \& Hansson, J. (2013). How implementation of bibliometric practice affects the role of academic libraries. Journal of Librarianship and Information Science, 45(4), 316-322. doi: http://doi.org/10.1177/0961000612456867

Ball, R., \& Tunger, D. (2006). Bibliometric analysis - A new business area for information professionals in libraries? Scientometrics, 66(3), 561-577. doi: http://doi.org/10.1007/s11192-006-0041-0

Chinchilla-Rodríguez, Z. (2004). Análisis del dominio científico español: 1995- 2002 (Tesis de doctorado). Universidad de Granada, Granada.

Corrall, S., Kennan, M. A., Afzal, W. \& Sen, B. (2013). Bibliometrics and Research Data Management Services: Emerging Trends in Library Support for Research. Library Trends, 61(3), 636-674. doi: http://doi.org/10.1353/lib.2013.0005

Glänzel, W. (2012). Bibliometrics in a nutshell. ESSS 2013. ESSS, Berlín. 
Iribarren-Maestro, I., Grandal, T., Alecha, M., Nieva, A., \& San-Julián, T. (2015). Apoyando la investigación: nuevos roles en el servicio de bibliotecas de la Universidad de Navarra. El profesional de la información, 24(2), 131-137.

Johnson, L., Butler, T. \& Johnston, L. (2012). Developing E-Science and Research Services and Support at the University of Minnesota Health Sciences Libraries. Journal of Library Administration, 52(8), 754-769. DOI:

http://doi.org/10.1080/01930826.2012.751291

Maceviciute, E. (2014). Research libraries in a modern environment. Journal of Documentation, 70(2), 282-302. doi: http://doi.org/10.1108/JD-04-2013-0044

Pinto. M., Sales, D. \& Osorio, P. (2008). Biblioteca universitaria, CRAl y alfabetización informacional. España: Ediciones Trea.

Rueda-Clausen Gómez, C. F., Villa-Roel, C., \& Rueda-Clausen Pinzón, C. E. (2005). Indicadores bibliométricos: origen, aplicación, contradicción y nuevas propuestas. MedUNAB, 8(1), 29-36.

Santos Jiménez, M., Cabrales Hernández, G., Rojas Mesa, Y., Gregorio Chaviano, O., \& Ramírez Mirabal, R. (2002). Análisis de los actuales servicios de información para los centros de investigación. Ciência da Informação, 31(2), 52-59.

Torres-Salinas, D., \& Cabezas-Clavijo, Á. (2012). Herramientas para la evaluación de la ciencia en universidades y centros I+D: descripción y usos. Anuario ThinkEPI, 6, 142-146.

Torres-Salinas, D., \& Jiménez-Contreras, E. (2012). Hacia las unidades de bibliometría en las universidades: modelo y funciones. Revista Española de Documentación Científica, 35(3), 469-480. doi: http://doi.org/10.3989/redc.2012.3.959

Tovar-Sanz, M. R. (2015). El apoyo a la investigación en las bibliotecas universitarias españolas. Documentación de las Ciencias de la Información, 38, 311-326. doi: http://dx.doi.org/10.5209/rev DCIN.2015.v38.50822

Villaveces, J., Bonilla, R., Bucheli, A., Chavarro, D., Delgado, L., Montilla, C Zarama, R. (2010). La investigación en Uniandes: construcción de una política. Bogotá: Universidad de los Andes, Vicerrectoría de Investigaciones, Ediciones univesitarias. 


\section{Datos de los autores}

Eliana Camila Arciniegas Tinjacá

Profesional en Ciencia de la Información y Bibliotecología de la Pontificia Universidad Javeriana - Bogotá. Gestor de Servicios de Información, Sistema de Bibliotecas - Universidad de los Andes

ec.arciniegas@uniandes.edu.co

\section{Yury Marcela Gómez Gutiérrez}

Profesional en Ciencia de la Información y Bibliotecología de la Pontificia Universidad Javeriana - Bogotá. Gestor de Servicios de Aprendizaje e Investigación, Sistema de Bibliotecas - Universidad de los Andes ym.gomez24@uniandes.edu.co

\section{Orlando Gregorio-Chaviano}

Doctorando en Comunicación. Máster en Información y Comunicación Científica. Profesor del Departamento de Ciencia de la Información. Facultad de Comunicación y Lenguaje. Pontificia Universidad Javeriana. Bogotá, Colombia.

ogregorio@javeriana.edu.co

Recibido - Received: 2017-03-27

Aceitado - Accepted: 2018-02-12

\section{(c) $)$ EY}

This work is licensed under a Creative Commons Attribution 4.0

United States License.

\section{ULIS DDof}

This journal is published by the University Library System of the University of Pittsburgh as part of its D-Scribe Digital Publishing Program and is cosponsored by the University of Pittsburgh Press. 